\title{
Community Based Rehabilitation after Neurologic Injury
}

Each year 1.7 million people incur a brain injury, nearly 800,000 suffer a stroke, and 12,000 endure a spinal cord injury. While there is often dramatic and early recovery, individuals with neurologic disabilities are often faced with long-lasting effects that continue well beyond acute hospitalization and inpatient rehabilitation, if any. After initial hospitalization, there is often a lengthy period of readjustment in which individuals must learn a new way of life. Disability often impacts activities of daily living, employment, housing, and a variety health issues. Individuals with the most support often have the best outcomes. The problem is, with decreasing lengths of stay in the hospital, limited financial resources for ongoing rehabilitation and other continuing needs, how can we best support individuals with neurological disabilities in their adjustment and return to productive living in the community?

While systems and services may already be in place within the formal healthcare community to assist those with neurologic disability, once the individual transitions outside the formal healthcare community, it can be very difficult to obtain information and access resources. There are many resources available, but not all resources are going to work for every person. It is often left to each individual to take the initiative to find and access appropriate programs and resources. Frequently, much time and effort is wasted with duplicative and incorrectly focused endeavors. Continued care often falls to the family and other community members. In the 1980's, community based rehabilitation became the vehicle for continued recovery once individuals left the formal healthcare system. According to the World Health Organization, community based rehabilitation is implemented through the combined efforts of people with disabilities, their families, organizations and communities, relevant government and non-government health, education, vocational, social and other services.
This particular issue of NeuroRehabilitation focuses on multiple areas of community based rehabilitation for individuals with neurologic disabilities. Martelli and colleagues kick off the issue with an overview of community rehabilitation in which they help define and classify the developing field of community rehabilitation as a whole, propose concepts for best practices, and propose methodologies for treatment. The following three articles cover specific issues that could impact an individual's ability to even return to the community including vision difficulties, information processing, and behavioral problems. Once in the community, rehabilitation efforts often focus on return to work, school, and productive living. The following two articles provide an overview of vocational rehabilitation models in general and an example of a community-based mentoring program for returning individuals with neurologic disability to productive activity.

In addition to in the individual with the disability, rehabilitation professionals must also consider the ongoing care needs of the individual's support system; particularly the family. Taryn Stejskal provides information on a theory-based approach to community based family intervention. Finally, as described earlier, neurologic disability is a life-long process. The final article of this issue discusses ongoing medical and general health-related consequences as they relate to lifetime management of disability.

We hope you enjoy the issue.

\footnotetext{
Stephanie A. Kolakowsky-Hayner, PhD, CBIST Director of Rehabilitation Research Santa Clara Valley Medical Center 751 S. Bacom Avenue San Jose, CA 95128, USA Tel.: +1 408793 6446; Fax: +1 4087936434 E-mail: Stephanie.Hayner@hhs.sccgov.org
} 